\title{
Des précisions sur l'interaction du produit du gène $R b$ avec des oncogènes
}

Le gène $\mathrm{Rb}$ de susceptibilité au rétinoblastome code pour une protéine d'environ 105000 daltons (les estimations varient en fait de 105 à 114 $\mathrm{kDa}$ que nous désignerons ici par $\ll \mathrm{p} 105^{\mathrm{Rb}} »$. Cette protéine forme des complexes stables avec les produits d'oncogènes nucléaires viraux tels $\mathrm{E}_{\mathrm{l}} \mathrm{A}$ d'adénovirus et l'antigène $\mathrm{T}$ de SV $40\left(\mathrm{~m} / \mathrm{s} n^{\circ} 8\right.$, vol. 4 , p. 520 et $n^{\circ} 10$, vol. 4, p. 606). $\mathrm{Pl} 105^{\mathrm{Rb}}$ aussi bien que T de SV40 sont des phosphoprotéines. L'équipe de De Caprio et Livingston (Dana-Farber Cancer institute et Harvard medical school, Boston, MA, USA) vient de montrer que c'était la forme déphosphorylée (ou sous-phosphorylée) de pl05 qui complexait préférentiellement l'antigène $T[1]$, ce qui suggère que le pouvoir antiprolifératif de cette protéine pourrait être modulé par phosphorylation-déphosphorylation.

- L'examen des immunoprécipités obtenus avec un anticorps anti $\mathrm{E}_{1} \mathrm{~A}$ révèle, outre $\mathrm{E}_{1} \mathrm{~A}$ et $\mathrm{pl} 05^{\mathrm{Rb}}$, des protéines de $300 \mathrm{kDa}$ et de $107 \mathrm{kDa}$ [2]. P. Whyte, du laboratoire de EdHarlow (Cold Spring Harbor Laboratory, New York, USA) a analysé les acides aminés de la protéine $\mathrm{E}_{1} \mathrm{~A}$ nécessaires à ces interactions. p300 et pl07 semblent interagir avec les résidus 1 à 76 et 121 à 127 , alors que $\mathrm{pl} 05^{\mathrm{Rb}}$ interagit avec les résidus 30 à 60 et 121 à 127 . Les modifications de $\mathrm{E}_{1} \mathrm{~A}$ dans ces régions, même celles qui n'empêchent pas la fixation de pl05 $5^{\mathrm{Rb}}$, suppriment son pouvoir transformant, ce qui suggère que l'interaction avec p300 et pl07 est également indispensable à la cancérisation cellulaire. Ces deux protéines sont de bons candidats pour correspondre aux produits d'oncogènes encore inconnus.

$\mathrm{m} / \mathrm{s} n^{\circ} 4$ vol. 5 , avril 89
- Il existe une lignée cellulaire, dérivée d'un cancer de vessis, dans laquelle la protéine $\mathrm{E}_{1} \mathrm{~A}$ n'interagit pas avec le produit du gène $R b$ qui $\mathrm{a}$ ici un poids moléculaire légèrement diminué (102000 aulieu de $105000)$. Les laboratoires de RA Weinberg (Whitehead, MIT, Cambridge MA, USA), Ed Harlow et le département d'ophtalmologie de T.P. Dryga (Harvard medical school, Boston, MA, USA) ont démontré que cette anomalie était secondaire à une mutation de la séquence «consensus » AG à l'extrémité 3' d'un intron, entraînant l'excision anormale de l'exon adjacent du transcrit et la production d'un messager et d'une protéine tronqués [3]. Peut-être peut-on imaginer que cette anomalie inhibe la complexation d'oncogènes, tel $\mathrm{E}_{1} \mathrm{~A}$, qui restent ainsi libres et disponibles pour activer la prolifération cellulaire.

- Ni les adénovirus, ni SV40 ne semblent impliqués dans l'apparition de cancers humains. C'est dire l'importance des travaux de l'équipe de Ed. Harlow qui a démontré que la protéine E7 des papillomavirus, équivalent très probable $\mathrm{d}^{\prime} \mathrm{E}_{1} \mathrm{~A}$ d'adénovirus, formait elle aussi un complexe stable avec p105 $\mathrm{Rb}$ [4]. Les sérotypes 16 et 18 des papillomavirus humains sont à l'origine d'une proportion très importante des cancers du col de l'utérus $\left(\mathrm{m} / \mathrm{s} n^{\circ} 7\right.$, vol. 2 , p. 405) et les résultats de Harlow suggèrent l'un des mécanismes possibles de cette carcinogenèse. Si le gène $R b$ est vraiment impliqué dans ce processus, on pourrait cependant s'attendre à ce que certains de ces cancers soient associés à son inactivation, comme cela a été montré (outre, évidemment, le rétinoblastome) dans des cancers du sein, du poumon et des tumeurs mésenchymateuses $\left(\mathrm{m} / \mathrm{s} n^{\circ} 8\right.$, vol. 4 , p. 520). De tous ces résultats émerge une confirmation : la prolifération cellulaire est bien contrôlée par un équilibre entre des substances la stimulant et d'autres l'inhibant. Des ambiguïtés persistent cependant sur les mécanismes d'action respectifs des oncogènes et des anti-oncogènes nucléaires. Des mutations d' $E_{1} A$ et de T de SV40 qui altèrent leur action propre sur l'expression d'autres gènes (et, pour T, sa liaison à l'ADN) ne suppriment pas leur pouvoir transformant dès lors qu'ils restent capables de se complexer avec pl05 $5^{\mathrm{Rb}}$ [5], ce qui suggère un modèle dans lequel le produit du gène $R b$ contrôle, à l'état libre, l'activité de gènes dont l'action contribue à inhibiber la prolifération cellulaire (activation de gènes inhibiteurs ou inhibition de gènes activateurs); les oncogènes nucléaires entraîneraient une «titration» de pl05 $5^{\mathrm{Rb}}$ qui ne serait ainsi plus disponible pour exercer son rôle inhibiteur et n'auraient, par eux-mêmes, pas d'action stimulatrice de la prolifération. Cependant, l'observation d'une mutation du gène $R b$, supprimant son interaction avec des oncogènes dans une lignée cancéreuse interpelle les spécialistes : dans ce cas, en effet, les oncogènes ont, justement, perdu leur pouvoir de «titrer» le produit du gène $R b$ qui, de plus, a gardé ses propriétés de liaison à l'ADN (l'exon excisé ne contient pas les structures en doigts contactant probablement l'ADN). A ce stade, deux explications peuvent être proposées : soit, en fait, les oncogènes nucléaires libres jouent un rôle propre dans la carcinogenèse; soit, l'exon excisé de l'ARNm Rb anormal code pour une

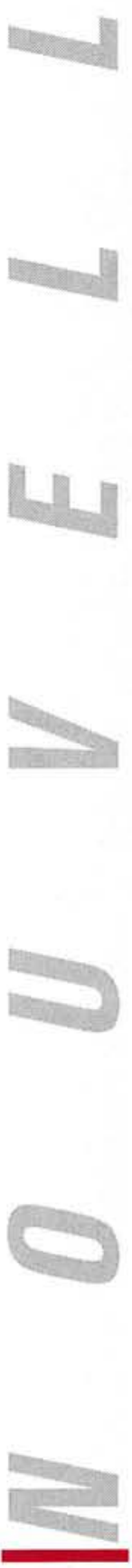


structure peptidique qui est impliquée à la fois dans la fixation des oncogènes et dans le contrôle de l'activation de gènes cibles, cibles encore parfaitement inconnues. On peut même supposer que, normalement, les complexes oncogènes - pl05 n'ont pas perdu leur propriété de liaison à l'ADN mais, une foisfixés, n'excercent plus l'influence modulatrice de $\mathrm{pl} 05^{\mathrm{Rb}}$ seule; une mutation de la zone d'interaction avec les oncogènes aurait le même effet que son «blocage » par ces mêmes oncogènes. A noter que nous parlons dans les lignes qui précèdent de l'interaction du produit du gène du rétinoblas$\mathrm{t}$ o m e a vec les «o n cog èn es nucléaires »... alors qu'elle n'a, à ce jour, été démontrée qu'avec ceux codés pardes virus oncogènes à $\mathrm{ADN}$ et dont il n'existe pas d'équivalent dans les génomes animaux. Qu'en est-il des produits des oncogènes nucléaires endogènes tels $m y c, m y b$, fos, jun? Nul doute que la réponse sera apportée très prochainement. Fos et Jun sont des activateurs transcriptionnels avérés de gènes répondant à la stimulation de la prolifération cellulaire, si bien que leur éventuel interaction avec $\mathrm{pl} 05^{\mathrm{Rb}}$ pourrait bien avoir la double signification d'une modulation mutuelle de substances jouant, par elles-même, pour certaines un rôle activateur et pour d'autres un rôle inhibiteur de la prolifération. Tout excès d'oncogènes aboutirait à la présence d'activateurs libres, tout excès de pl05 $5^{\mathrm{Rb}}$ aboutissant à la présence de molécules inhibitrices libres.

A.K.

1. ludlow JW, De Caprio JA, Huang CM, Lee $W \cdot H$, Paucha E, Livingston DM. SV40 large $T$ antigen binds preferentially to an underphosphorylated member of the retinoblastoma susceptibility gene product family. Cell $1989 ; 56: 57-65$.

2. Whyte P, Williamson NM, Harlow E. Cellular targets for transformation by the adenovirus ElA proteins. Cell $1989 ; 56: 67-75$.

3. Horowitz JM, Yandell DW, Park SH, et al. Point mutational inactivation of the retinoblastoma antioncogene. Science 1989; 243 : 937-40.

4. Dyson N, Howley PM, Münger K, Harlow E. The human papilloma virus-16 E7 oncoprotein is able to bind to the retinoblastoma gene product. Science 1989 ; 243 : 934-7.

5 . Green MR. When the products of oncogenes and antioncogenes meet. Cell 1989; 56

De l'extérieur de la membrane plasmique au noyau, il existe de multiples étapes par lesquelles passent les «messages» de prolifération cellulaire. Un dérèglement à l'une ou à l'autre de ces étapes peut conduire à la cancérisation. De même qu'il est possible, pratiquement, de décrire des oncogènes caractéristiques de chacun des stades de la transmission d'un signal prolifératif au noyau cellulaire, existe-t-il, à tous ces niveaux, des produits d'anti-oncogènes exercant un contrôle négatif? On connaît déjà le gène de susceptibilité au rétinoblastome dont le produit, nucléaire, interagit avec les produits d'oncogènes nucléaires (voir nouvelle précédente). Très récemment, de nouveaux anti-oncogènes présomptifs ont été isolés, et il est probable que le mouvement est appelé à se développer. La stratégie utilisée par le groupe de M. Noda (Tsukuba, Japon) a été d'introduire dans des cellules tumorales (transformées par l'oncogène $v$ Kirsten-ras) des ADNc de fibroblastes humains clonés dans un vecteur d'expression. Des clones ayant perdu l'essentiel de leur tumorigénicité ont été isolés. Chacun de ces clones semble spécifiquement résistant à la surtransformation par un groupe d'oncogènes donné. L'ADNc de l'un de ces clones a été caractérisé en détail. Il se révèle en effet capable d'entraîner une reversion des cellules transformées vers un phénotype peu ou pas tumorigène [1], résistant à la surtransformation par des oncogènes de type $v$-ras, mais non $v$-src, $v$-mas, $v$ - raf, ou v-fos. La séquence nucléotidique révèle que ce clone, appellé Krev-1, code pour une protéine de 21 $\mathrm{kDa}$ à $50 \%$ analogue aux $\mathrm{p} 2 \mathrm{l}^{\text {ras }}$. Krev-1 est identique au produit du gène rap-1 isolé par V. Pizon et al. (laboratoire d'Armand Tavitian, INSERM, Paris) [2] sur la base d'analogies de séquence avec les gènes de la famille ras. Rappelons que les protéines de la famille ras sont des molécules liant le GTP, dont on pense qu'elles interviennent dans la transduction d'un signal prolifératif, encore que l'on ne connaisse pas encore le récepteur et le système effecteur qu'elles coupleraient. Quoiqu'il en soit, les protéines p2l ras rappellent beaucoup les sous-unités $\alpha$ des $G$ protéines, par exemple de celles couplées à l'adénylate cyclase. Il existe des sous-unités $\alpha_{\mathrm{s}}$ (stimulatrices) et $\alpha_{\mathrm{i}}$ (inhibitrices). De même, existe-t-il des $\mathrm{p} 2 \mathrm{I}_{\mathrm{s}}^{\mathrm{ras}}$ (les produits des gènes $\mathrm{Ha}$ ras, $K i$-ras, $N$-ras) et des p2l ras (le produit du gène Krev-1/rap-1) ?La nature nous a habitué à ce que les processus clés de la vie soient tous contrôlés à la fois par des systèmes positifs et par des systèmes négatifs. Si cela est applicable (et comment cela pourrait-il ne pas l'être!) au contrôle de la division cellulaire, il faut nous attendre à ce que soient. décrits des produits d'anti-oncogènes agissant aux mêmes niveaux que les produits d'oncogènes. On connaît déjà des substances diffusibles inhibitrices de croissance (TGF $\beta$, hormone antimüllerienne, peut-être les interférons, $\mathrm{m} / \mathrm{s} \mathrm{n}^{\circ} 8$, vol. 2, p. 467) 